Comparison of spread spectrum and pulse signal excitation for split spectrum techniques composite imaging

This article has been downloaded from IOPscience. Please scroll down to see the full text article.

2012 IOP Conf. Ser.: Mater. Sci. Eng. 42012007

(http://iopscience.iop.org/1757-899X/42/1/012007)

View the table of contents for this issue, or go to the journal homepage for more

Download details:

IP Address: 158.42.249.4

The article was downloaded on 22/03/2013 at 16:00

Please note that terms and conditions apply. 


\title{
Comparison of spread spectrum and pulse signal excitation for split spectrum techniques composite imaging
}

\author{
L Svilainis ${ }^{1}$, K Kitov $^{1}$, A Rodríguez ${ }^{2}$, L Vergara ${ }^{3}$, V Dumbrava ${ }^{1}$, and A \\ Chaziachmetovas ${ }^{1}$ \\ ${ }^{1}$ Kaunas University of Technology, Department of Signal Processing Studentu str. 50- \\ 340, LT-51368, Kaunas, Lithuania \\ ${ }^{2}$ Universidad Miguel Hernández de Elche, Department of Communications \\ Engineering, Avda. Universidad S/N, 03202 Elche, Spain \\ ${ }^{3}$ Universidad Politécnica de Valencia, Department of Communications, Camino de \\ Vera S/N, 46022 Valencia, Spain \\ E-mail: linas.svilainis@ktu.lt
}

\begin{abstract}
Ultrasonic imaging of composites was investigated. Glass and carbon fiber reinforced plastic produced by resin transfer molding and prepreg forming were analyzed. In some of the samples air bubbles were trapped during RTM (resin transfer molding) process and interlayer gaps were present in prepreg technology samples. One of the most expected techniques to apply in such case is the Split Spectrum processing. On the other hand such signals require specific processing to reliably reconstruct the temporal position of the defect reflection. Correlation processing can be used for signal compression or Wiener filtering can be applied for spectral content equalisation. Pulse signals are simple to generate, but lack the possibility to alter the signal's spectrum shape. Spread spectrum signals offer a powerful tool for signal energy over frequency band increase and resolution enhancement. CW (continuous wave) burst has high energy but lacks the bandwidth needed for SSP (spread spectrum processing). The aim of the investigation was to compare the performance of the above signals in case of composite imaging, when various Split Spectrum Processing techniques are used with preceding Wiener processing for spectral content compensation. Resulting composite signals and images obtained are presented. Structural noise removal performance was evaluated as Receiver Operating Characteristics (ROC).
\end{abstract}

\section{Introduction}

Ultrasonic imaging of composite materials can not use conventional techniques since signals scattered by composite structure are masking the useful signal [1]. Due to the presence of the structural noise, resolving the defect or even the backwall of the material is complicated.

Techniques used for composite imaging can be divided into two categories: i) using the frequency range least affected by the structure (usually low frequencies); ii) techniques exploring the diversity of the structure signals so averaging can be applied. Using low frequencies is not always acceptable since resolution of imaging depends on envelope bandwidth which usually is a direct function of operation frequency. Techniques exploring the diversity can be further divided into spatial (synthetic aperture focusing technique, SAFT [1]) and frequency domain (Split Spectrum Processing, SSP [2]). 
We have concentrated on SSP technique. Assumption was made that SSP processing calls for smooth and as wide as possible signal bandwidth. Then, we aimed our investigation on Spread Spectrum (SS) signals performance comparison with conventional excitation signals when applied in SSP technique.

\section{Split Spectrum Processing}

SSP algorithm has been around for decades [2] and is considered as sub-optimal to the problem of detection of the ultrasonic pulse in a structural noise [3-8]. Technique is based on the assumption that the echoes coming from the scattering centers of the material poses diversity in frequency domain, i.e., the structure noise response is expected to vary significantly at different frequency channels. The echo from a large discontinuity, on the contrary, will act the same way in different channels. Hence, most of the SSP techniques implement a filter bank followed by a combination of the filter outputs (figure 1).

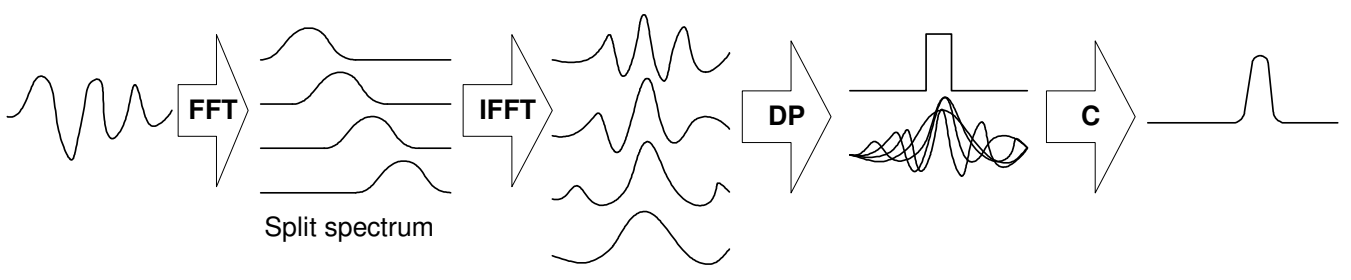

Figure 1. Standard data flow diagram for Split Spectrum Processing.

Main combination techniques used in our investigation were: [4] polarity thresholding (PT), [6] scaled polarity thresholding (SPT), [8] frequency multiplication (FM) and [3] minimization (MIN). Minimisation was chosen as a classical reference, its output is a minimum value of the filter banks. In PT, if polarity of the bank signals is the same, useful signal is assumed and average of the banks output is used, if at least one bank polarity does not match, zero mask is used. SPT is essentially the same as PT, only it uses linear mask, produced by ratio of same $\left(N_{+}\right)$and opposite polarity $\left(N_{-}\right)$banks to total banks number $N$. FM is a kind of geometric mean, where product of banks output is used.

\subsection{Performance measures}

Signals performance measurement was done using the Receiver Operating Characteristics (ROC). We have assumed that the goal of the SSP is the lowest probability of the false alarm (PFA) while maintaining maximum of the probability of detection (PD). PFA and PD were obtained as false alarm counts $N_{\mathrm{FA}}$ and detection counts $N_{\mathrm{D}}$ at variable threshold level $V_{\text {th }}$ normalized to possible events $\mathrm{N}$ :

$$
\operatorname{PFA}\left(V_{t h}\right)=\frac{N_{F A}\left(V_{t h}\right)}{N} \quad P D\left(V_{t h}\right)=\frac{N_{D}\left(V_{t h}\right)}{N}
$$

Obtained PFA and PD were plotted against each other to produce ROC [9].

\section{System setup}

System (figure 2) is composed from control part, excitation pulser and the signals acquisition units. Industrial standard PC104 modular construction was used as form factors and bus. The excitation pulser driver has $100 \mathrm{MHz}$ sampling rate and is driving the totem pole topology pulser [10]. Code upload and control is accomplished by host PC via PC104 bus. The receiving part is preceded by high impedance preamplifier [11] with high voltage protection input limiter. Conditioned signal is supplied to the dual high speed ADC, consisting of two high speed 10-bit monolithic sampling ADC operating at programmable sampling frequency up to $100 \mathrm{MS} / \mathrm{s}$. All the control tasks and PC104 bridge functions are implemented by Complex Programmable Logic Device (CPLD) chip. 

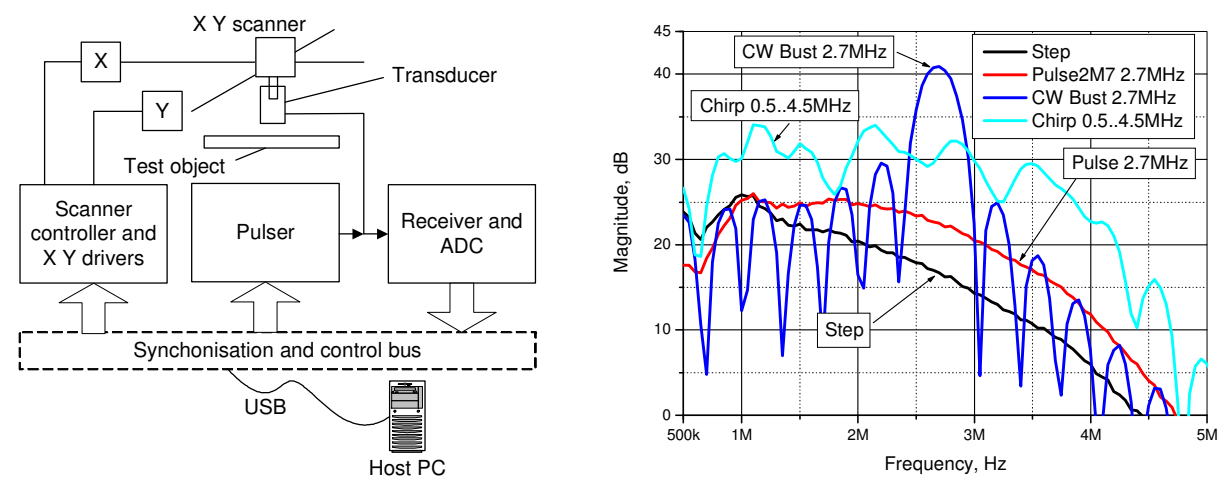

Figure 2. System setup (left) and spectral content of excitation signals used (right).

Five signals were uploaded into pulser memory for subsequent excitation: $\mathrm{CW}$ burst of $1 \mathrm{MHz}$ frequency, CW burst of $2.7 \mathrm{MHz}$ frequency (transducer center frequency), $0.5 \mathrm{MHz}$ to $4.5 \mathrm{MHz}$ spread spectrum signal implemented as linear chirp, rectangular pulse with duration $270 \mathrm{~ns}$ (corresponds to $2.7 \mathrm{MHz}$ transducer center frequency), $3 \mathrm{~ns}$ steep step corresponding to frequency range up to $106 \mathrm{MHz}$. We deliberately avoided techniques exploring the phase correlation [7] since these techniques should be less sensitive to the amplitude variation among the banks. Since spread spectrum signals were used, SSP algorithm was modified, adding the Wiener processing [4] before SSP to compensate the spectral content and concentrate all components into one temporal position.

\section{Experimental results}

Three materials were used in investigation: thin $(2.2 \mathrm{~mm})$ sample of kayak wall, sputtered GFRP (glass fiber reinforced plastic) composite; thick $(4.9 \mathrm{~mm})$ multilayer sample of kayak wall: GFRP plus random carbon filament plus glass fiber net; and high porosity GFRP, $9 \mathrm{~mm}$ thick: randomly oriented chipped glass fibers. Spherically focused ultrasonic transducer, TS 12PB2-7P30, diameter $12 \mathrm{~mm}$, $1.5 \mathrm{~mm}$ focal spot was chosen for its narrow beam. Transducer was operated with focal spot positioned on material under investigation. In such case spatial averaging is minimal and effect of the structural noise is maximised. Resulting B-scan images for multilayer kayak are presented in figure 3.



Figure 3. B-scan image obtained at various signal and combination techniques: multilayer kayak. 

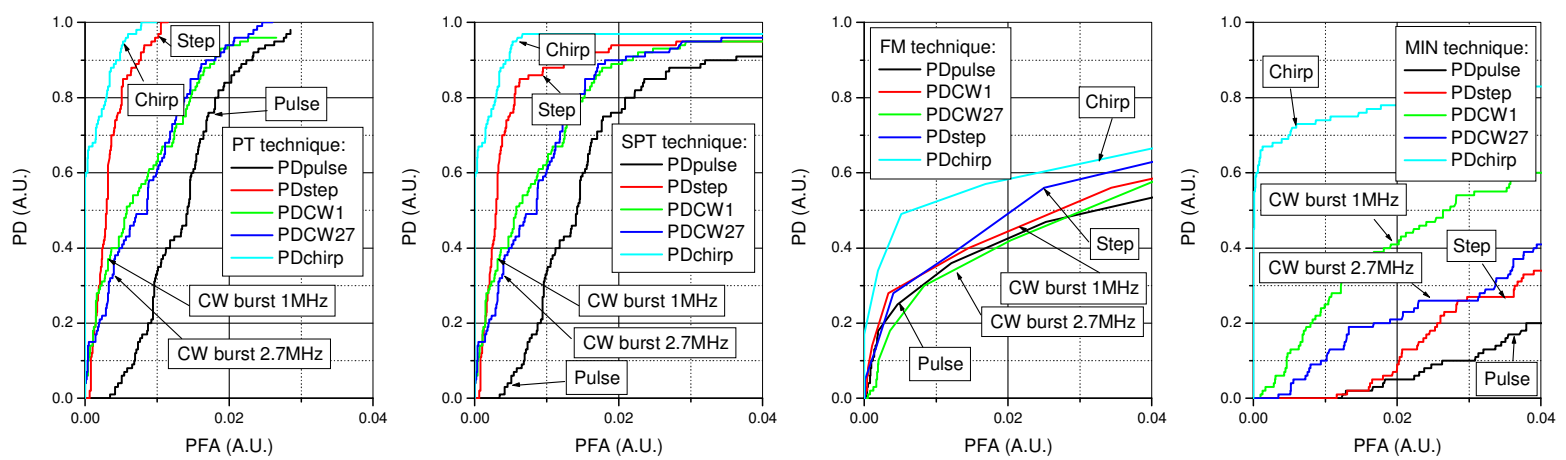

Figure 4. Signals' performance comparison: ROC for high porosity composite.

ROCs for the excitation signals used for high porosity composite imaging are presented in figure 4. Investigation indicates that best performance is obtained for spread spectrum (chirp) signal. Signal performs best in polarity thresholding SSP processing.

\section{Conclusions}

Use of spread spectrum excitation gives best performance for majority of materials and techniques. Best performance was achieved in conjunction with rectangular mask techniques in combination with minimization. Pulse and step excitation signals performance was comparable, though at narrower choice of techniques. CW burst signals sometimes are falsely assumed as good performers if duration after correlation compression is not taken into account. In some cases, when reflection contained not all frequency components, signals with larger low frequency content were in favor.

\section{Acknowledgments}

This research (acquisition system and spread spectrum signals) was funded by a grant (No. MIP058/2012) from the Research Council of Lithuania. SSP part was supported by PROMETEO 2010/40.

\section{References}

[1] Li Y, Newhouse V L, Shankar P M, Karpur P 1992 Speckle reduction in ultrasonic synthetic aperture images Ultrasonics 30 223-237

[2] Bilgutay N M, Saniie J, Furgason E S, and Newhouse V L 1979 Flaw-to-grain echo enhansment. Proc.Ultrasonics International (Austria, May 1979) p 157

[3] Karpur P, Shankar P, Rose J and Newhouse V L 1987 Split spectrum processing: optimising the processing parameters using minimisation. Ultrasonics 25204

[4] Saffari N, Svilainis L 1997 Signal processing for ultrasonic imaging of lossy composites Ultragarsas 28 7-11

[5] Ericsson L, Stepinski T 2002 Algorithms for suppressing ultrasonic backscattering from material structure Ultrasonics 40 733-734.

[6] Rubbers P, Pritchard C J 2003 An overview of split spectrum processing NDT.net 88.

[7] Rubbers P and Pritchard C 2003 Complex plane Split Spectrum Processing: an introduction NDT.net $\mathbf{8} 11$

[8] Rodríguez A, Miralles R, Bosch I, Vergara L 2012 New analysis and extensions of splitspectrum processing algorithms NDT and E International 45 141-147

[9] Rodríguez A, Salazar A, Vergara L, 2012 Analysis of split-spectrum algorithms in an automatic detectionframework Signal Processing 92 2293-2307

[10] Svilainis L, Chaziachmetovas A, Kybartas D, Jurkonis R 2011 Advanced Hardware for FineTuning and Optimization of Sonoporation Efficiency In vitro Proc. IDAACS (Prague,15-17 September 2011) pp 65-70

[11] Svilainis L, Dumbrava V, Chaziachmetovas A 2010 Ultrasonic Preamplifier Performance Evaluation Proc. IEEE ITI (Croatia, 21-24 June 2010) vol 32 pp 663 - 668 